\title{
Extensions of some matrix inequalities related to trace and partial traces*
}

\author{
Yongtao $\mathrm{Li}^{*}$ \\ School of Mathematics, Hunan University \\ Changsha, Hunan, 410082, P.R. China
}

January 20, 2022

Dedicated to Prof. Weijun Liu on his 60th birthday

\begin{abstract}
We first present a determinant inequality related to partial traces for positive semidefinite block matrices. Our result extends a result of Lin [Czech. Math. J. 66 (2016)] and improves a result of Kuai [Linear Multilinear Algebra 66 (2018)]. Moreover, we provide a unified treatment of a result of Ando [ILAS Conference (2014)] and a recent result of Li, Liu and Huang [Operators and Matrices 15 (2021)]. Furthermore, we also extend some determinant inequalities involving partial traces to a larger class of matrices whose numerical ranges are contained in a sector. In addition, some extensions on trace inequalities for positive semidefinite $2 \times 2$ block matrices are also included.
\end{abstract}

Key words: Partial traces; Trace inequalities; Fiedler and Markham; Numerical range in a sector;

2010 Mathematics Subject Classification. 15A45, 15A60, 47B65.

\section{Introduction}

Throughout the paper, we use the following standard notation. The set of $n \times n$ complex matrices is denoted by $\mathbb{M}_{n}(\mathbb{C})$, or simply by $\mathbb{M}_{n}$, and the identity matrix of order $n$ by $I_{n}$, or $I$ for short. We write $\lambda_{i}(A)$ and $\sigma_{i}(A)$ for the $i$-th largest eigenvalue and singular value of $A$, respectively. By convention, if $A \in \mathbb{M}_{n}$ is positive semidefinite, we write $A \geq 0$. For Hermitian matrices $A$ and $B$ with the same size,

*This paper was firstly announced in March, 2020, and was later published on Linear Algebra and its Applications 639 (2022) 205-224. See https://doi.org/10.1016/j.laa.2022.01.006. E-mail addresses: ytli0921@hnu.edu.cn (Yǒngtāo Li). 
$A \geq B$ means that $A-B$ is positive semidefinite, i.e., $A-B \geq 0$. If $A=\left[a_{i, j}\right]$ is of order $m \times n$ and $B$ is of order $s \times t$, the tensor product of $A$ with $B$, denoted by $A \otimes B$, is an $m s \times n t$ matrix that partitioned into $m \times n$ block matrices with the $(i, j)$-block being the $s \times t$ matrix $a_{i, j} B$. In this paper, we are interested in complex block matrices. Let $\mathbb{M}_{n}\left(\mathbb{M}_{k}\right)$ be the set of complex matrices partitioned into $n \times n$ blocks with each block being $k \times k$. The element of $\mathbb{M}_{n}\left(\mathbb{M}_{k}\right)$ is usually written as $H=\left[H_{i, j}\right]_{i, j=1}^{n}$, where $H_{i, j} \in \mathbb{M}_{k}$ for all $i, j$.

Now we introduce the definition of partial traces, which comes from Quantum Information Theory [32, p. 12]. For $H \in \mathbb{M}_{n}\left(\mathbb{M}_{k}\right)$, the first partial trace (map) $H \mapsto \operatorname{tr}_{1} H \in \mathbb{M}_{k}$ is defined as the adjoint map of the embedding map $X \mapsto I_{n} \otimes X \in$ $\mathbb{M}_{n} \otimes \mathbb{M}_{k}$. Correspondingly, the second partial trace (map) $H \mapsto \operatorname{tr}_{2} H \in \mathbb{M}_{n}$ is defined as the adjoint map of the embedding map $Y \mapsto Y \otimes I_{k} \in \mathbb{M}_{n} \otimes \mathbb{M}_{k}$. Therefore, we have

$$
\left\langle I_{n} \otimes X, H\right\rangle=\left\langle X, \operatorname{tr}_{1} H\right\rangle, \quad \forall X \in \mathbb{M}_{k},
$$

and

$$
\left\langle Y \otimes I_{k}, H\right\rangle=\left\langle Y, \operatorname{tr}_{2} H\right\rangle, \quad \forall Y \in \mathbb{M}_{n},
$$

where $\langle\cdot, \cdot\rangle$ stands for the Hilbert-Schmidt inner product, i.e., $\langle A, B\rangle=\operatorname{tr}\left(A^{*} B\right)$. The above definition of partial traces is implicit. Assume that $H=\left[H_{i, j}\right]_{i, j=1}^{n}$ is an $n \times n$ block matrix with $H_{i, j} \in \mathbb{M}_{k}$, the visualized version of the partial traces is equivalently given in [4. pp. 120-123] as

$$
\operatorname{tr}_{1} H=\sum_{i=1}^{n} H_{i, i}
$$

and

$$
\operatorname{tr}_{2} H=\left[\operatorname{tr} H_{i, j}\right]_{i, j=1}^{n} .
$$

It is easy to see that both $\operatorname{tr}_{1} H$ and $\operatorname{tr}_{2} H$ are positive semidefinite whenever $H \in$ $\mathbb{M}_{n}\left(\mathbb{M}_{k}\right)$ is positive semidefinite; see, e.g. [36, p. 237] or [37] for more details. The first or second partial trace is a source for matrix inequalities and extensively studied in recent years; see [2, 7, 11, 22, 29, for related topics.

Let $A=\left[A_{i, j}\right]_{i, j=1}^{n}$ be an $n \times n$ block matrix with each block being a $k \times k$ matrix. The usual transpose of $A$ is defined as $A^{T}=\left[A_{j, i}^{T}\right]_{i, j=1}^{n}$. We define the partial transpose of $A$ by $A^{\tau}=\left[A_{j, i}\right]_{i, j=1}^{n}$, that is, the partial transpose of $A$ is the matrix obtained by transposing blocks of $A$ independently. More precisely,

$$
A^{T}=\left[\begin{array}{ccc}
A_{1,1}^{T} & \cdots & A_{n, 1}^{T} \\
\vdots & \ddots & \vdots \\
A_{1, n}^{T} & \cdots & A_{n, n}^{T}
\end{array}\right] \text { and } A^{\tau}=\left[\begin{array}{ccc}
A_{1,1} & \cdots & A_{n, 1} \\
\vdots & \ddots & \vdots \\
A_{1, n} & \cdots & A_{n, n}
\end{array}\right]
$$

Although $A$ and $A^{\tau}$ have the same trace, they may have different eigenvalues, so they are not necessarily similar. Moreover, it is known that $A \geq 0$ does not necessarily 
imply $A^{\tau} \geq 0$. For example, taking

$$
A=\left[\begin{array}{ll}
A_{1,1} & A_{1,2} \\
A_{2,1} & A_{2,2}
\end{array}\right]=\left[\begin{array}{cc:cc}
1 & 0 & 0 & 1 \\
0 & 0 & 0 & 0 \\
\hdashline 0 & 0 & 0 & 0 \\
1 & 0 & 0 & 1
\end{array}\right]
$$

We can see from the definition that

$$
A^{\tau}=\left[\begin{array}{ll}
A_{1,1} & A_{2,1} \\
A_{1,2} & A_{2,2}
\end{array}\right]=\left[\begin{array}{cc:cc}
1 & 0 & 0 & 0 \\
0 & 0 & 1 & 0 \\
\hdashline 0 & 1 & 0 & 0 \\
0 & 0 & 0 & 1
\end{array}\right] .
$$

One could easily observe that $A$ is positive semidefinite, but $A^{\tau}$ is not positive semidefinite since it contains a principal submatrix $\left[\begin{array}{ll}0 & 1 \\ 1 & 0\end{array}\right] \ngtr 0$. Moreover, the eigenvalues of $A$ are $2,0,0,0$, and the eigenvalues of $A^{\tau}$ are $1,1,1,-1$, so $A$ and $A^{\tau}$ are not similar. In addition, replacing $A_{1,1}$ in the above matrix by $\left[\begin{array}{ll}1 & 0 \\ 0 & 1\end{array}\right]$ also gives a well example. From this discussion, we say that $A$ is positive partial transpose (or PPT for short) if both $A$ and $A^{\tau}$ are positive semidefinite. We recommend [10, 19, 26, 27] for recent progress.

The paper is organized as follows. In Section 2, we shall review some preliminaries for a class of matrices whose numerical ranges are contained in a sector (known as the sector matrices). This is a natural extension of the class of positive definite matrices. In Section 3, we shall study the recent results involving the Fiedler-Markham inequality. We provide an extension of a result of Lin [29], and our result is also an improvement of a result of Kuai [17; see Theorem [3.5. Moreover, we shall extend a result of Choi [6] to the so-called sector matrices; see Theorem 3.7. In Section 4, we give a unified treatment of a result of Ando [2] (or see [30]) as well as a recent result of Li, Liu and Huang [22]. Our new treatment is more concise than original proof. Moreover, we also present some Ando type determinant inequalities for partial traces, and then we extend these inequalities to sector matrices; see Theorems 4.7 and 4.8 . In Section 5, we shall prove some inequalities for positive semidefinite $2 \times 2$ block matrices; see Theorems 5.2, 5.3 and 5.4. Our result extend slightly the recent elegant work on trace inequalities that proved by Kittaneh and Lin [18] and Lin [26] as well.

\section{Preliminaries}

Recall that $\sigma_{i}(A)$ denotes $i$-th largest singular value of $A$. When $A$ is Hermitian, we know that all eigenvalues of $A$ are real numbers, and we write $\lambda_{i}(A)$ for the $i$-th largest eigenvalue. The numerical range of $A \in \mathbb{M}_{n}$ is defined by

$$
W(A)=\left\{x^{*} A x: x \in \mathbb{C}^{n}, x^{*} x=1\right\} .
$$


For $\alpha \in[0, \pi / 2)$, let $S_{\alpha}$ be the sector on complex plane defined as

$$
S_{\alpha}=\{z \in \mathbb{C}: \Re z>0,|\Im z| \leq(\Re z) \tan \alpha\}=\left\{r e^{i \theta}: r>0,|\theta| \leq \alpha\right\} .
$$

For $A \in \mathbb{M}_{n}$, the Cartesian (Toeptliz) decomposition is given as $A=\Re A+i \cdot \Im A$, where

$$
\Re A=\frac{1}{2}\left(A+A^{*}\right) \text { and } \Im A=\frac{1}{2 i}\left(A-A^{*}\right) .
$$

We know from the definition that if $W(A) \subseteq S_{0}$, then $A$ is positive definite. Moreover, it is easy to verify that if $W(A) \subseteq S_{\alpha}$ for some $\alpha \in[0, \pi / 2)$, then $\Re(A)$ is positive definite. Such class of matrices whose numerical ranges are contained in a sector is called the sector matrices class. Clearly, the concept of sector matrices is an extension of positive definite matrices. Over the past few years, various studies on sector matrices have been obtained in the literature; see, e.g., [8, 16, 17, 28, 34, 38].

Before starting our results, we now summarise the following lemmas.

Lemma 2.1 [28] Let $0 \leq \alpha<\pi / 2$ and $A \in \mathbb{M}_{n}$ with $W(A) \subseteq S_{\alpha}$. Then

$$
|\operatorname{det} A| \leq(\sec \alpha)^{n} \operatorname{det}(\Re A) \text {. }
$$

Lemma 2.2 [14, p. 510] Let $X$ be an n-square complex matrix. Then

$$
\lambda_{i}(\Re X) \leq \sigma_{i}(X), \quad i=1,2, \ldots, n .
$$

Moreover, if $\Re X$ is positive definite, then

$$
\operatorname{det} \Re X+|\operatorname{det} \Im X| \leq|\operatorname{det} X|
$$

The following lemma is called the Fischer inequality, which gives an upper bound for the determinant of a positive semidefinite block matrix in terms of the determinants of its principal diagonal blocks. In particular, when all blocks have order $1 \times 1$, this inequality is also known as the Hadamard inequality; see, e.g., [14, p. 506] and [36, p. 217].

Lemma 2.3 Let $H=\left[H_{i, j}\right]_{i, j=1}^{n} \in \mathbb{M}_{n}\left(\mathbb{M}_{k}\right)$ be positive semidefinite. Then

$$
\operatorname{det} H \leq \prod_{i=1}^{n} \operatorname{det} H_{i, i} .
$$

Lemma 2.4 If $H \in \mathbb{M}_{n}\left(\mathbb{M}_{k}\right)$ satisfies $W(H) \subseteq S_{\alpha}$, then $W\left(\operatorname{tr}_{1} H\right) \subseteq S_{\alpha}$ and $W\left(\operatorname{tr}_{2} H\right) \subseteq$ $S_{\alpha}$, i.e., if $H$ is a sector matrix with angle $\alpha \in[0, \pi / 2)$, then so are $\operatorname{tr}_{1} H$ and $\operatorname{tr}_{2} H$. 
We remark that this lemma was partially proved in [17, Proposition 3.2] for the case $\operatorname{tr}_{2} H$. Motivated by [17], we here include a detailed proof for the remaining case $\operatorname{tr}_{1} H$.

Proof. Consider the Cartesian decomposition $H=\Re H+i \cdot \Im H$, then

$$
\operatorname{tr}_{1} H=\operatorname{tr}_{1}(\Re H)+i \cdot \operatorname{tr}_{1}(\Im H) .
$$

For every $x \in \mathbb{C}^{k}$ with $x^{*} x=1$, as $\Re H$ is positive definite, we get

$$
\Re\left(x^{*}\left(\operatorname{tr}_{1} H\right) x\right)=x^{*}\left(\Re\left(\operatorname{tr}_{1} H\right)\right) x=x^{*}\left(\operatorname{tr}_{1}(\Re H)\right) x>0 .
$$

On the other hand, by a direct computation,

$$
\frac{\left|\Im\left(x^{*}\left(\operatorname{tr}_{1} H\right) x\right)\right|}{\Re\left(x^{*}\left(\operatorname{tr}_{1} H\right) x\right)}=\frac{\left|x^{*}\left(\operatorname{tr}_{1}(\Im H)\right) x\right|}{x^{*}\left(\operatorname{tr}_{1}(\Re H)\right) x}=\frac{\left|\left\langle x x^{*}, \operatorname{tr}_{1}(\Im H)\right\rangle\right|}{\left\langle x x^{*}, \operatorname{tr}_{1}(\Re H)\right\rangle} .
$$

Note that $I_{n} \otimes\left(x x^{*}\right)$ is positive semidefinite. We consider the spectral decomposition

$$
I_{n} \otimes\left(x x^{*}\right)=\sum_{i=1}^{n k} \lambda_{i} u_{i} u_{i}^{*}
$$

where $\lambda_{i} \geq 0$ and $u_{i}$ are unit vectors in $\mathbb{C}^{n k}$. By the definition in (1), it follows that

$$
\begin{aligned}
\frac{\left|\left\langle x x^{*}, \operatorname{tr}_{1}(\Im H)\right\rangle\right|}{\left\langle x x^{*}, \operatorname{tr}_{1}(\Re H)\right\rangle} & =\frac{\left|\left\langle I_{n} \otimes\left(x x^{*}\right), \Im H\right\rangle\right|}{\left\langle I_{n} \otimes\left(x x^{*}\right), \Re H\right\rangle}=\frac{\left|\sum_{i=1}^{n k} \lambda_{i}\left\langle u_{i} u_{i}^{*}, \Im H\right\rangle\right|}{\sum_{i=1}^{n k} \lambda_{i}\left\langle u_{i} u_{i}^{*}, \Re H\right\rangle} \\
& \leq \frac{\sum_{i=1}^{n k} \lambda_{i}\left|u_{i}^{*}(\Im H) u_{i}\right|}{\sum_{i=1}^{n k} \lambda_{i} u_{i}^{*}(\Re H) u_{i}} \leq \max _{1 \leq i \leq n k} \frac{\left|u_{i}^{*}(\Im H) u_{i}\right|}{u_{i}^{*}(\Re H) u_{i}}=\max _{1 \leq i \leq n k} \frac{\left|\Im\left(u_{i}^{*} H u_{i}\right)\right|}{\Re\left(u_{i}^{*} H u_{i}\right)} .
\end{aligned}
$$

This completes the proof.

Remark. Based on the second equivalent definition (2), one could also give other ways to prove Lemma 2.4. We leave the details for the interested reader.

\section{Extensions on Fiedler-Markham's inequality}

Let $H=\left[H_{i, j}\right]_{i, j=1}^{n} \in \mathbb{M}_{n}\left(\mathbb{M}_{k}\right)$ be positive semidefinite. Recall that both $\operatorname{tr}_{1} H$ and $\operatorname{tr}_{2} H$ are positive semidefinite; see, e.g., [37]. In 1994, Fiedler and Markham [9, Corollary 1] proved a celebrated determinant inequality involving the second partial trace.

Theorem 3.1 [9] Let $H=\left[H_{i, j}\right]_{i, j=1}^{n} \in \mathbb{M}_{n}\left(\mathbb{M}_{k}\right)$ be positive semidefinite. Then

$$
\left(\frac{\operatorname{det}\left(\operatorname{tr}_{2} H\right)}{k}\right)^{k} \geq \operatorname{det} H
$$


In 2016, Lin [29] revisited this inequality using some terminology from quantum information theory, and gave an alternative proof of Theorem 3.1 by applying an important identity connecting $\operatorname{tr}_{2} H$ and $H$. Moreover, a natural question is that whether an analogous result corresponding to the Fiedler-Markham inequality holds for $\operatorname{tr}_{1} H$. Lin [29] answered this question and proved the following counterpart.

Theorem 3.2 [29] Let $H=\left[H_{i j}\right]_{i, j=1}^{n} \in \mathbb{M}_{n}\left(\mathbb{M}_{k}\right)$ be positive semidefinite. Then

$$
\left(\frac{\operatorname{det}\left(\operatorname{tr}_{1} H\right)}{n}\right)^{n} \geq \operatorname{det} H \text {. }
$$

It is clear that in the proof of both Theorem 3.1 and Theorem 3.2 , Fiedler and Markham, and Lin used the superadditivity of determinant functional, which states that

$$
\operatorname{det}\left(\sum_{i=1}^{n} H_{i, i}\right) \geq \sum_{i=1}^{n} \operatorname{det} H_{i, i} \geq n\left(\prod_{i=1}^{n} \operatorname{det} H_{i, i}\right)^{1 / n} .
$$

This inequality can be improved by the Fan-Ky determinant inequality (see [14, p. $488]$ ), i.e., the log-concavity of the determinant over the cone of positive semidefinite matrices:

$$
\operatorname{det}\left(\frac{1}{n} \sum_{i=1}^{n} H_{i, i}\right) \geq\left(\prod_{i=1}^{n} \operatorname{det} H_{i, i}\right)^{1 / n} .
$$

In addition, we mention here that a careful examination of the new proof of Theorem 3.1 in [29] can also reveal this improvement. This improvement was also pointed out in [6, 31]. Next, we state the strong version of Theorem 3.1 and Theorem 3.2.

Theorem 3.3 Let $H=\left[H_{i j}\right]_{i, j=1}^{n} \in \mathbb{M}_{n}\left(\mathbb{M}_{k}\right)$ be positive semidefinite. Then

$$
\left(\frac{\operatorname{det}\left(\operatorname{tr}_{2} H\right)}{k^{n}}\right)^{k} \geq \operatorname{det} H
$$

and

$$
\left(\frac{\operatorname{det}\left(\operatorname{tr}_{1} H\right)}{n^{k}}\right)^{n} \geq \operatorname{det} H
$$

We observe in Theorem 3.3 that the second inequality seems easier to prove than the first inequality because it is more convenient to build inequalities on $\operatorname{tr}_{1} H=$ $\sum_{i=1}^{n} H_{i, i}$. In [20], the authors showed that both inequalities can be deduced mutually.

In 2018, Kuai [17] (or see [34]) further extended Theorem 3.3 to sector matrices and showed that if $0 \leq \alpha<\pi / 2$ and $H \in \mathbb{M}_{n}\left(\mathbb{M}_{k}\right)$ satisfies $W(H) \subseteq S_{\alpha}$, then

$$
\left|\frac{\operatorname{det}\left(\operatorname{tr}_{2} H\right)}{k^{n}}\right|^{k} \geq(\cos \alpha)^{n k}|\operatorname{det} H|
$$


and

$$
\left|\frac{\operatorname{det}\left(\operatorname{tr}_{1} H\right)}{n}\right|^{n} \geq(\cos \alpha)^{(3 n-2) k}|\operatorname{det} H| .
$$

Our first goal in this section is to improve Kuai's result (6). The key step in our improvement is the following identity connecting $\operatorname{tr}_{1}(H)$ and $H$, which has been applied to quantum information theory, such as the sub-additivity of $q$-entropies. This identity can be found in [15, eq.(26)] or [5, Lemma 2].

Lemma 3.4 Let $X$ and $Y$ be generalized Pauli matrices on $\mathbb{C}^{n}$; these operators act as $X e_{j}=e_{j+1}$ and $Y e_{j}=e^{2 \pi j \sqrt{-1} / n} e_{j}$, where $e_{j}$ is the $j$-th column of the identity matrix $I_{n}$ and $e_{n+1}=e_{1}$. Then

$$
\frac{1}{n} \sum_{l, j=1}^{n}\left(X^{l} Y^{j} \otimes I_{k}\right) H\left(X^{l} Y^{j} \otimes I_{k}\right)^{*}=I_{n} \otimes\left(\operatorname{tr}_{1} H\right) .
$$

Remark. The identity in this lemma can yield an alternative proof of Lemma 2.4. Moreover, the analogous identity for $\operatorname{tr}_{2} H$ can be seen in [15] or [33, eq.(14)].

Now, we are ready to present an improvement on inequality (6).

Theorem 3.5 Let $0 \leq \alpha<\pi / 2$ and $H \in \mathbb{M}_{n}\left(\mathbb{M}_{k}\right)$ be such that $W(H) \subseteq S_{\alpha}$. Then

$$
\left|\frac{\operatorname{det}\left(\operatorname{tr}_{1} H\right)}{n^{k}}\right|^{n} \geq(\cos \alpha)^{n k}|\operatorname{det} H| \text {. }
$$

Proof. Note that both $X$ and $Y$ in Lemma 3.4 are unitary, so are $X^{l} Y^{j} \otimes I_{k}$ for all $l, j$. Moreover, we have $\Re\left(U H U^{*}\right)=U(\Re H) U^{*}$ for every unitary $U$. Thus,

$$
\begin{aligned}
& |\operatorname{det} H|=\prod_{l, j=1}^{n}\left|\operatorname{det}\left(X^{l} Y^{j} \otimes I_{k}\right) H\left(X^{l} Y^{j} \otimes I_{k}\right)^{*}\right|^{1 / n^{2}} \\
& \stackrel{\text { Lemma }}{\leq .11}(\sec \alpha)^{n k} \prod_{l, j=1}^{n}\left(\operatorname{det}\left(X^{l} Y^{j} \otimes I_{k}\right)(\Re H)\left(X^{l} Y^{j} \otimes I_{k}\right)^{*}\right)^{1 / n^{2}} \\
& \stackrel{\text { Fan-Ky ineq. (4) }}{\leq}(\sec \alpha)^{n k} \operatorname{det}\left(\frac{1}{n^{2}} \sum_{l, j=1}^{n}\left(X^{l} Y^{j} \otimes I_{k}\right)(\Re H)\left(X^{l} Y^{j} \otimes I_{k}\right)^{*}\right)
\end{aligned}
$$

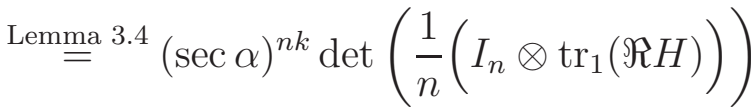

$$
\begin{aligned}
& =\frac{(\sec \alpha)^{n k}}{n^{n k}} \operatorname{det}\left(I_{n} \otimes \operatorname{tr}_{1}(\Re H)\right) .
\end{aligned}
$$

Clearly, we have $\operatorname{tr}_{1}(\Re H)=\Re\left(\operatorname{tr}_{1} H\right)$. For $X \in \mathbb{M}_{n}$ and $Y \in \mathbb{M}_{k}$, it is well-known that $\operatorname{det}(X \otimes Y)=(\operatorname{det} X)^{k}(\operatorname{det} Y)^{n}$; see, e.g., [35, Chapter 2]. It follows that

$$
\operatorname{det}\left(I_{n} \otimes \operatorname{tr}_{1}(\Re H)\right)=\left(\operatorname{det} I_{n}\right)^{k}\left(\operatorname{det}\left(\operatorname{tr}_{1} \Re H\right)\right)^{n}=\left(\operatorname{det} \Re\left(\operatorname{tr}_{1} H\right)\right)^{n} .
$$


By Proposition 2.4, we have $W\left(\operatorname{tr}_{1} H\right) \subseteq S_{\alpha}$, which implies that $\Re\left(\operatorname{tr}_{1} H\right)$ is positive definite. Therefore, by Lemma 2.2, we get

$$
\left(\operatorname{det} \Re\left(\operatorname{tr}_{1} H\right)\right)^{n} \leq\left(\left|\operatorname{det}\left(\operatorname{tr}_{1} H\right)\right|-\left|\operatorname{det} \Im\left(\operatorname{tr}_{1} H\right)\right|\right)^{n} \leq\left|\operatorname{det}\left(\operatorname{tr}_{1} H\right)\right|^{n},
$$

which together with (77) yields the desired result.

Remark. By applying the techniques from [20], we know that Kuai's inequality (5) can also be deduced from the inequality in Theorem 3.5 and vice versa.

In the sequel, we shall focus our attention on some recent results which are similar with the Fiedler-Markham inequality. Let $H=\left[H_{i, j}\right]_{i, j=1}^{n} \in \mathbb{M}_{n}\left(\mathbb{M}_{k}\right)$ be a block matrix with $H_{i, j}=\left[h_{l, m}^{i, j}\right]_{l, m=1}^{k}$. We define an $n \times n$ matrix $G_{l, m}$ as below.

$$
G_{l, m}:=\left[h_{l, m}^{i, j}\right]_{i, j=1}^{n} \in \mathbb{M}_{n}
$$

A direct computation yields

$$
\operatorname{tr}_{1} H=\sum_{i=1}^{n} H_{i, i}=\sum_{i=1}^{n}\left[h_{l, m}^{i, i}\right]_{l, m=1}^{k}=\left[\sum_{i=1}^{n} h_{l, m}^{i, i}\right]_{l, m=1}^{k}=\left[\operatorname{tr} G_{l, m}\right]_{l, m=1}^{k} .
$$

For notational convenience, we denote

$$
\widetilde{H}=\left[G_{l, m}\right]_{l, m=1}^{k} \in \mathbb{M}_{k}\left(\mathbb{M}_{n}\right)
$$

We can see that $\widetilde{H}$ is obtained from $H$ by rearranging the entries in an appropriate order. The above observation yields $\operatorname{tr}_{1} H=\operatorname{tr}_{2} \widetilde{H}$. Moreover, it is not hard to check that $\widetilde{H}$ and $H$ are unitarily similar; see, e.g., [6, Theorem 7] or [20, Theorem 4]. Motivated by these relations, Choi [6] introduced recently the definition of partial determinants corresponding to partial traces. For $H=\left[H_{i, j}\right]_{i, j=1}^{n} \in \mathbb{M}_{n}\left(\mathbb{M}_{k}\right)$, the partial determinants are defined as

$$
\operatorname{det}_{1} H:=\left[\operatorname{det} G_{l, m}\right]_{l, m=1}^{k},
$$

and

$$
\operatorname{det}_{2} H:=\left[\operatorname{det} H_{i, j}\right]_{i, j=1}^{n} .
$$

To some extent, the partial determinants share some common properties relative to partial traces. For instance, it is easy to see that if $H \in \mathbb{M}_{n}\left(\mathbb{M}_{k}\right)$ is positive semidefinite, then both $\operatorname{det}_{1} H$ and $\operatorname{det}_{2} H$ are positive semidefinite; see, e.g. [36, p. 221]. Moreover, it was proved in [6] that

$$
\operatorname{det}\left(\operatorname{tr}_{1} H\right) \geq \operatorname{tr}\left(\operatorname{det}_{2} H\right)
$$

and

$$
\operatorname{det}\left(\operatorname{tr}_{2} H\right) \geq \operatorname{tr}\left(\operatorname{det}_{1} H\right)
$$

Additionally, Choi [6] proved two analogues of Theorem 3.1] and Theorem [3.2 for partial determinants. 
Theorem 3.6 [6] Let $H \in \mathbb{M}_{n}\left(\mathbb{M}_{k}\right)$ be positive semidefinite. Then

$$
\left(\frac{\operatorname{tr}\left(\operatorname{det}_{1} H\right)}{k}\right)^{k} \geq \operatorname{det} H
$$

and

$$
\left(\frac{\operatorname{tr}\left(\operatorname{det}_{2} H\right)}{n}\right)^{n} \geq \operatorname{det} H
$$

Next, we will extend Theorem 3.6 to sector matrices. We write $|A|$ for the nonnegative matrix whose entries are the absolute of the entries of $A$. This notation is only used in the following theorem.

Theorem 3.7 Let $0 \leq \alpha<\pi / 2$ and $H \in \mathbb{M}_{n}\left(\mathbb{M}_{k}\right)$ be such that $W(H) \subseteq S_{\alpha}$. Then

$$
\left(\frac{\operatorname{tr}\left|\operatorname{det}_{1} H\right|}{k}\right)^{k} \geq(\cos \alpha)^{n k}|\operatorname{det} H|,
$$

and

$$
\left(\frac{\operatorname{tr}\left|\operatorname{det}_{2} H\right|}{n}\right)^{n} \geq(\cos \alpha)^{n k}|\operatorname{det} H|
$$

Proof. First of all, we shall prove the second inequality. We observe that $\Re H_{1,1}$, $\ldots, \Re H_{n, n}$ are the diagonal block matrices of $\Re H$. By Lemma 2.1 and Lemma 2.3, we obtain

$$
\begin{aligned}
|\operatorname{det} H| & \leq(\sec \alpha)^{n k} \operatorname{det}(\Re H) \leq(\sec \alpha)^{n k} \prod_{i=1}^{n} \operatorname{det}\left(\Re H_{i, i}\right) \\
& \leq(\sec \alpha)^{n k} \prod_{i=1}^{n}\left|\operatorname{det} H_{i, i}\right| \leq(\sec \alpha)^{n k}\left(\frac{1}{n} \sum_{i=1}^{n}\left|\operatorname{det} H_{i, i}\right|\right)^{n},
\end{aligned}
$$

where the third inequality follows from Lemma 2.2 and the last one follows from the arithmetic mean-geometric mean inequality.

We now prove the first desired inequality by employing the relations between $\operatorname{det}_{1}$ and $\operatorname{det}_{2}$. Recall that $\widetilde{H}=\left[G_{l, m}\right]_{l, m=1}^{k} \in \mathbb{M}_{k}\left(\mathbb{M}_{n}\right)$ and $\operatorname{det}_{1} H=\operatorname{det}_{2} \widetilde{H}$. Since $\widetilde{H}$ and $H$ are unitarily similar, we can get $\operatorname{det} \widetilde{H}=\operatorname{det} H$ and $W(\widetilde{H}) \subseteq S_{\alpha}$. Moreover, $\widetilde{H}$ is also positive semidefinite. By applying the second inequality to $\widetilde{H}$, we get

$$
\left(\frac{\operatorname{tr}\left|\operatorname{det}_{1} H\right|}{k}\right)^{k}=\left(\frac{\operatorname{tr}\left|\operatorname{det}_{2} \widetilde{H}\right|}{k}\right)^{k} \geq(\cos \alpha)^{k n}|\operatorname{det} \widetilde{H}|=(\cos \alpha)^{k n}|\operatorname{det} H| .
$$

This completes the proof. 


\section{Extensions on Ando's inequality}

To make our statements more transparent and compatible with the previous works in the literature. In this section, we assume that $A$ is an $m \times m$ block matrix with each block being an $n \times n$ matrix. Let $A=\left[A_{i, j}\right]_{i, j=1}^{m} \in \mathbb{M}_{m}\left(\mathbb{M}_{n}\right)$ be positive semidefinite. We know that both $\operatorname{tr}_{1} A$ and $\operatorname{tr}_{2} A$ are positive semidefinite; see, e.g., [36, p. 237] and [37, Theorem 2.1]. To some degree, these two partial traces are closely related and

mutually affect each other. We write $\|A\|_{q}=\left(\sum_{i} \sigma_{i}(A)^{q}\right)^{1 / q}$ for the Schatten $q$-norm of $A$. In 2007, Audenaert [1] proved the following norm inequality,

$$
\operatorname{tr} A+\|A\|_{q} \geq\left\|\operatorname{tr}_{1} A\right\|_{q}+\left\|\operatorname{tr}_{2} A\right\|_{q}
$$

A straightforward argument exploiting Audenaert's result leads to a proof of the subadditivity of $q$-entropies (Tsallis entropies) for finite-dimensional bipartite quantum states; see [1, 5] and references therein. In 2014, Ando [2] (or see [30, Proposition 2.2] for an alternative proof) established the following remarkable inequality in the sense of the Löwner ordering.

Theorem 4.1 [2, 30] Let $A \in \mathbb{M}_{m}\left(\mathbb{M}_{n}\right)$ be positive semidefinite. Then

$$
(\operatorname{tr} A) I_{m n}+A \geq I_{m} \otimes\left(\operatorname{tr}_{1} A\right)+\left(\operatorname{tr}_{2} A\right) \otimes I_{n}
$$

Ando's result reveals closely the interplay between the first and second partial trace. Equivalently, this inequality can be rewritten as

$$
(\operatorname{tr} A) I_{m n}-\left(\operatorname{tr}_{2} A\right) \otimes I_{n} \geq I_{m} \otimes\left(\operatorname{tr}_{1} A\right)-A
$$

We observe that the positivity of $A$, together with the identity $\operatorname{tr} A=\sum_{i=1}^{m} \operatorname{tr} A_{i, i}=$ $\operatorname{tr}\left(\operatorname{tr}_{2} A\right)$, leads to $(\operatorname{tr} A) I_{m} \geq \lambda_{\max }\left(\operatorname{tr}_{2} A\right) I_{m} \geq \operatorname{tr}_{2} A$, which guarantees that in (9) the left hand side $(\operatorname{tr} A) I_{m n}-\left(\operatorname{tr}_{2} A\right) \otimes I_{n}$ is positive semidefinite. However, the two matrices of the right hand side in (9) might be incomparable. For instance, the matrix $A$ in (3) gives an example. Motivated by this observation, Li, Liu and Huang [22] presented a further generalization.

Theorem 4.2 [22] Let $A \in \mathbb{M}_{m}\left(\mathbb{M}_{n}\right)$ be positive semidefinite. Then

$$
(\operatorname{tr} A) I_{m n}-\left(\operatorname{tr}_{2} A\right) \otimes I_{n} \geq A-I_{m} \otimes\left(\operatorname{tr}_{1} A\right)
$$

and

$$
(\operatorname{tr} A) I_{m n}+\left(\operatorname{tr}_{2} A\right) \otimes I_{n} \geq A+I_{m} \otimes\left(\operatorname{tr}_{1} A\right)
$$

A map (not necessarily linear) $\Phi: \mathbb{M}_{n} \rightarrow \mathbb{M}_{k}$ is called positive if it maps positive semidefinite matrices to positive semidefinite matrices. A map $\Phi: \mathbb{M}_{n} \rightarrow \mathbb{M}_{k}$ is said to be $m$-positive if for every $m \times m$ block matrix $\left[A_{i, j}\right]_{i, j=1}^{m} \in \mathbb{M}_{m}\left(\mathbb{M}_{n}\right)$,

$$
\left[A_{i, j}\right]_{i, j=1}^{m} \geq 0 \Rightarrow\left[\Phi\left(A_{i, j}\right)\right]_{i, j=1}^{m} \geq 0
$$


Clearly, being 1-positive is equivalent to being positive. The map $\Phi$ is said to be completely positive if it is $m$-positive for every integer $m \geq 1$. It is well-known that both the trace map and determinant map are completely positive; see, e.g., [36, p. 221 , p. 237] or [37]. On the other hand, a map $\Phi$ is said to be $m$-copositive if for every $\left[A_{i, j}\right]_{i, j=1}^{m} \in \mathbb{M}_{m}\left(\mathbb{M}_{n}\right)$,

$$
\left[A_{i, j}\right]_{i, j=1}^{m} \geq 0 \Rightarrow\left[\Phi\left(A_{j, i}\right)\right]_{i, j=1}^{m} \geq 0
$$

and $\Phi$ is said to be completely copositive if it is $m$-copositive for every integer $m \geq 1$. Furthermore, a map $\Phi$ is called completely PPT if it is both completely positive and completely copositive; see [26, 10, 39] for related topics.

Both Theorem 4.1 and Theorem 4.2 illustrated the implicit interaction and connection between the first trace and second trace. The proof of Theorem 4.1 depends mainly on the 2-copositivity of $\Psi(X)=(\operatorname{tr} X) I-X$; see e.g., [2] and [30] for more details. Correspondingly, the proof of Theorem 4.2 relies similarly on the 2-copositivity of $\Phi(X)=(\operatorname{tr} X) I+X$; see [22]. For more application of these two maps, we refer readers to papers [26, 21].

In this section, we give a unified treatment of both Theorem 4.1 and Theorem 4.2. Our treatment is more concise than the original proof. We need to use a recent result of Choi [6, 7], which investigates more relations between the partial traces and the partial transpose.

Lemma 4.3 [6, 17] Let $A \in \mathbb{M}_{m}\left(\mathbb{M}_{n}\right)$ be positive semidefinite. Then

$$
\left(\operatorname{tr}_{2} A^{\tau}\right) \otimes I_{n} \geq \pm A^{\tau}
$$

and

$$
I_{m} \otimes \operatorname{tr}_{1} A^{\tau} \geq \pm A^{\tau}
$$

Now, we present a unified treatment of Theorems 4.1 and 4.2 as well.

New proof of Theorem 4.1. We define the map $\Phi: \mathbb{M}_{m}\left(\mathbb{M}_{n}\right) \rightarrow \mathbb{M}_{m}\left(\mathbb{M}_{n}\right)$ as

$$
\Phi_{2}^{-}(X):=\left(\operatorname{tr}_{2} X^{\tau}\right) \otimes I_{n}-X^{\tau} .
$$

On the other hand, we define

$$
\Phi_{1}^{-}(X):=I_{m} \otimes \operatorname{tr}_{1} X^{\tau}-X^{\tau} .
$$

Lemma 4.3 implies that both $\Phi_{2}^{-}$and $\Phi_{1}^{-}$are positive linear maps on $\mathbb{M}_{m}\left(\mathbb{M}_{n}\right)$. Let $A$ be a positive semidefinite block matrix. Thus, we have

$$
\Phi_{2}^{-}(A)=\left(\operatorname{tr}_{2} A^{\tau}\right) \otimes I_{n}-A^{\tau} \geq 0 .
$$

Acting the map $\Phi_{1}^{-}$to the matrix $\Phi_{2}^{-}(A)$, we can obtain

$$
\Phi_{1}^{-}\left(\Phi_{2}^{-}(A)\right)=I_{m} \otimes \operatorname{tr}_{1} \Phi_{2}^{-}(A)^{\tau}-\Phi_{2}^{-}(A)^{\tau} \geq 0 .
$$


By a directed computation, we can get $\Phi_{2}^{-}(A)^{\tau}=\left(\operatorname{tr}_{2} A\right) \otimes I_{n}-A$ and

$$
\operatorname{tr}_{1} \Phi_{2}^{-}(A)^{\tau}=\operatorname{tr}_{1}\left(\left(\operatorname{tr}_{2} A\right) \otimes I_{n}-A\right)=\sum_{i=1}^{m}\left(\operatorname{tr} A_{i, i}\right) I_{n}-\operatorname{tr}_{1} A=(\operatorname{tr} A) I_{n}-\operatorname{tr}_{1} A
$$

Therefore, inequality (10) yields the desired result in Theorem 4.1 .

Remarks. In the above proof, we can see that Theorem 4.1 is just a direct consequence of Lemma 4.3. To our surprise, Theorem 4.1 can also be proved by using the positivity of $\Phi_{1}^{-}$first, and then applying the positivity of $\Phi_{2}^{-}$later. More precisely, we first derive $\Phi_{1}^{-}(A) \geq 0$, and then we have $\Phi_{2}^{-}\left(\Phi_{1}^{-}(A)\right) \geq 0$. Upon simplification, one can immediately get Theorem 4.1 again. We summarize this observation as the following proposition.

Proposition 4.4 For every $X \in \mathbb{M}_{m}\left(\mathbb{M}_{n}\right)$, we have $\Phi_{1}^{-}\left(\Phi_{2}^{-}(X)\right)=\Phi_{2}^{-}\left(\Phi_{1}^{-}(X)\right)$.

Correspondingly, we can present an alternative proof of Theorem 4.2 similarly.

New proof of Theorem 4.2. We define the maps $\Phi_{2}^{+}$and $\Phi_{1}^{+}$on $\mathbb{M}_{m}\left(\mathbb{M}_{n}\right)$ as

$$
\Phi_{2}^{+}(X):=\left(\operatorname{tr}_{2} X^{\tau}\right) \otimes I_{n}+X^{\tau},
$$

and

$$
\Phi_{1}^{+}(X):=I_{m} \otimes \operatorname{tr}_{1} X^{\tau}+X^{\tau} .
$$

We can see from Lemma 4.3 that both $\Phi_{2}^{+}$and $\Phi_{1}^{+}$are positive linear maps. Similar to the lines of the previous proof, we get $\Phi_{1}^{-}\left(\Phi_{2}^{+}(A)\right)=\Phi_{2}^{+}\left(\Phi_{1}^{-}(A)\right) \geq 0$, which leads to

$$
(\operatorname{tr} A) I_{m n}-\left(\operatorname{tr}_{2} A\right) \otimes I_{n} \geq A-I_{m} \otimes\left(\operatorname{tr}_{1} A\right) .
$$

Moreover, we have $\Phi_{1}^{+}\left(\Phi_{2}^{-}(A)\right)=\Phi_{2}^{-}\left(\Phi_{1}^{+}(A)\right) \geq 0$. It follows that

$$
(\operatorname{tr} A) I_{m n}+\left(\operatorname{tr}_{2} A\right) \otimes I_{n} \geq A+I_{m} \otimes\left(\operatorname{tr}_{1} A\right)
$$

We mention that the positivity of $\Phi_{1}^{+}\left(\Phi_{2}^{+}(A)\right)$ yields a trivial result.

In the remaining of this section, we shall pay attention to determinant inequalities of sector matrices involving partial traces. Motivated by Audenaert's result (8), Lin [29] recently obtained a determinantal inequality for partial traces, which states that if $A \in \mathbb{M}_{m}\left(\mathbb{M}_{n}\right)$ is positive semidefinite, then

$$
(\operatorname{tr} A)^{m n}+\operatorname{det} A \geq \operatorname{det}\left(\operatorname{tr}_{1} A\right)^{m}+\operatorname{det}\left(\operatorname{tr}_{2} A\right)^{n} .
$$

We remark here that $\mathrm{Fu}$, Lau and Tam [11, Corollary 2.2] recently improved (11) when $A$ is a density matrix, i.e., a positive semidefinite matrix with trace equal to 1 .

The key step in the proof of (11) attributes to Theorem 4.1 together with the following interesting lemma. It is worth noting that Lemma 4.5 is graceful and useful in deriving matrix inequalities; see, e.g., [23, 24, 25] for applications on Oppenheim type inequalities. 
Lemma 4.5 [30] Let $X, Y, W$ and $Z$ be positive semidefinite matrices of the same order. If $X \geq W, X \geq Z$ and $X+Y \geq W+Z$, then

$$
\operatorname{det} X+\operatorname{det} Y \geq \operatorname{det} W+\operatorname{det} Z \text {. }
$$

Remark. We observe that Lemma 4.5 implies the determinant inequality:

$$
\operatorname{det}(A+B+C)+\operatorname{det} C \geq \operatorname{det}(A+C)+\operatorname{det}(B+C),
$$

where $A, B$ and $C$ are positive semidefinite matrices.

With the help of Lemma 4.5, we can easily present two analogues of (11).

Proposition 4.6 Let $A \in \mathbb{M}_{m}\left(\mathbb{M}_{n}\right)$ be positive semidefinite. Then

$$
(\operatorname{tr} A)^{m n}+\operatorname{det}\left(\operatorname{tr}_{1} A\right)^{m} \geq \operatorname{det} A+\operatorname{det}\left(\operatorname{tr}_{2} A\right)^{n}
$$

and

$$
(\operatorname{tr} A)^{m n}+\operatorname{det}\left(\operatorname{tr}_{2} A\right)^{n} \geq \operatorname{det} A+\operatorname{det}\left(\operatorname{tr}_{1} A\right)^{m}
$$

Proof. We prove the first inequality only, since the second one can be proved in exactly the same way. Let $X=(\operatorname{tr} A) I_{m n}, Y=I_{m} \otimes\left(\operatorname{tr}_{1} A\right), W=A$ and $Z=$ $\left(\operatorname{tr}_{2} A\right) \otimes I_{n}$. It is easy to see that

$$
(\operatorname{tr} A) I_{m}=\sum_{i=1}^{m}\left(\operatorname{tr} A_{i, i}\right) I_{m}=\left(\operatorname{tr}\left(\operatorname{tr}_{2} A\right)\right) I_{m} \geq \lambda_{\max }\left(\operatorname{tr}_{2} A\right) I_{m} \geq \operatorname{tr}_{2} A,
$$

which implies that $X \geq Z \geq 0$, and clearly $X \geq W \geq 0$. Moreover, Theorem 4.2 says that $X+Y \geq W+Z$. That is, all conditions in Lemma 4.5 are satisfied. Therefore,

$$
(\operatorname{tr} A)^{m n}+\operatorname{det}\left(I_{m} \otimes\left(\operatorname{tr}_{1} A\right)\right) \geq \operatorname{det} A+\operatorname{det}\left(\left(\operatorname{tr}_{2} A\right) \otimes I_{n}\right)
$$

It is well-known [35, p. 37] that for every $X \in \mathbb{M}_{m}$ and $Y \in \mathbb{M}_{n}$,

$$
\operatorname{det}(X \otimes Y)=(\operatorname{det} X)^{n}(\operatorname{det} Y)^{m} .
$$

Thus, we complete the proof of the required result.

We next give an improvement on Proposition 4.6.

Theorem 4.7 Let $A \in \mathbb{M}_{m}\left(\mathbb{M}_{n}\right)$ be positive semidefinite. Then

$$
(\operatorname{tr} A)^{m n}+\operatorname{det}\left(\operatorname{tr}_{1} A\right)^{m} \geq m^{n m}\left(\operatorname{det} A+\operatorname{det}\left(\operatorname{tr}_{2} A\right)^{n}\right)
$$

and

$$
(\operatorname{tr} A)^{m n}+\operatorname{det}\left(\operatorname{tr}_{2} A\right)^{n} \geq n^{m n}\left(\operatorname{det} A+\operatorname{det}\left(\operatorname{tr}_{1} A\right)^{m}\right)
$$


Proof. We only prove the second inequality. Invoking Theorem 3.3, we get

$$
\left(\frac{\operatorname{det}\left(\operatorname{tr}_{2} A\right)}{n^{m}}\right)^{n} \geq \operatorname{det} A \text {. }
$$

Equivalently, we have $\operatorname{det}\left(\operatorname{tr}_{2} A\right)^{n} \geq n^{m n} \operatorname{det} A$. It suffices to show that

$$
(\operatorname{tr} A)^{n} \geq n^{n} \operatorname{det}\left(\operatorname{tr}_{1} A\right) .
$$

Note that

$$
\operatorname{tr} A=\sum_{i=1}^{m} \operatorname{tr}\left(A_{i, i}\right)=\operatorname{tr}\left(\sum_{i=1}^{m} A_{i, i}\right)=\operatorname{tr}\left(\operatorname{tr}_{1} A\right) .
$$

We denote $X:=\operatorname{tr}_{1} A$, which is a positive semidefinite matrix of order $n$. So we need to prove that $(\operatorname{tr} X)^{n} \geq n^{n} \operatorname{det} X$. This is equivalent to showing

$$
\left(\sum_{i=1}^{n} \lambda_{i}(X)\right)^{n} \geq n^{n} \prod_{i=1}^{n} \lambda_{i}(X)
$$

which is a direct consequence of the AM-GM inequality.

Surprisingly, the proof of Theorem 4.7 seems simpler than that of Proposition 4.6) since it does not rely on Theorem 4.2 and Lemma 4.5. However, it allows us to provide a great improvement on Proposition 4.6 whenever $m, n$ are large integers.

In the sequel, we shall denote $|A|=\left(A^{*} A\right)^{1 / 2}$, which is called the modulus of $A$. We remark that this notation is different from that in Theorem 3.7. Note that $|A|$ is positive semidefinite, and the eigenvalues of $|A|$ are called the singular values of $A$. In 2019, Yang, Lu and Chen [34] extended (11) to sector matrices.

$$
(\operatorname{tr}|A|)^{m n}+\operatorname{det}|A| \geq(\cos \alpha)^{m n}\left|\operatorname{det}\left(\operatorname{tr}_{1} A\right)\right|^{m}+(\cos \alpha)^{m n}\left|\operatorname{det}\left(\operatorname{tr}_{2} A\right)\right|^{n} .
$$

Now, we are ready to present an extension on Theorem 4.7 .

Theorem 4.8 Let $A \in \mathbb{M}_{m}\left(\mathbb{M}_{n}\right)$ be such that $W(A) \subseteq S_{\alpha}$. Then

$$
(\operatorname{tr}|A|)^{m n}+\left|\operatorname{det}\left(\operatorname{tr}_{1} A\right)\right|^{m} \geq(m \cos \alpha)^{m n}\left(\operatorname{det}|A|+\left|\operatorname{det}\left(\operatorname{tr}_{2} A\right)\right|^{n}\right),
$$

and

$$
(\operatorname{tr}|A|)^{m n}+\left|\operatorname{det}\left(\operatorname{tr}_{2} A\right)\right|^{n} \geq(n \cos \alpha)^{m n}\left(\operatorname{det}|A|+\left|\operatorname{det}\left(\operatorname{tr}_{1} A\right)\right|^{m}\right) .
$$

Proof. We only prove the first inequality. According to the definition of $S_{\alpha}$, if $W(A) \subseteq S_{\alpha}$, then $\Re A$ is positive definite and its trace is positive. By Lemma 2.2, we have

$$
\operatorname{tr}|A|=\sum_{i=1}^{m n} \sigma_{i}(A) \geq \sum_{i=1}^{m n} \lambda_{i}(\Re A)=\operatorname{tr}(\Re A) \geq 0 .
$$


It is noteworthy by Lemma 2.4 that $W\left(\operatorname{tr}_{1} A\right) \subseteq S_{\alpha}$ and $W\left(\operatorname{tr}_{2} A\right) \subseteq S_{\alpha}$. Clearly, we have $\Re\left(\operatorname{tr}_{1} A\right)=\operatorname{tr}_{1}(\Re A)$ and $\Re\left(\operatorname{tr}_{2} A\right)=\operatorname{tr}_{2}(\Re A)$. By setting $X=\operatorname{tr}_{1} A$ in Lemma 2.2 , we get

$$
\left|\operatorname{det}\left(\operatorname{tr}_{1} A\right)\right| \geq \operatorname{det}\left(\Re\left(\operatorname{tr}_{1} A\right)\right)=\operatorname{det}\left(\operatorname{tr}_{1}(\Re A)\right) .
$$

Note that $\Re A$ is positive semidefinite. By applying Theorem 4.7, we can obtain

$$
\begin{aligned}
(\operatorname{tr}|A|)^{m n}+\left|\operatorname{det}\left(\operatorname{tr}_{1} A\right)\right|^{m} & \geq(\operatorname{tr} \Re A)^{m n}+\left(\operatorname{det} \operatorname{tr}_{1}(\Re A)\right)^{m} \\
& \geq m^{n m}\left(\operatorname{det}(\Re A)+\left(\operatorname{det} \Re\left(\operatorname{tr}_{2} A\right)\right)^{n}\right) \\
& \geq(m \cos \alpha)^{m n}|\operatorname{det} A|+(m \cos \alpha)^{m n}\left|\operatorname{det}\left(\operatorname{tr}_{2} A\right)\right|^{n},
\end{aligned}
$$

where the last inequality holds from Lemma 2.2 by setting $X=A$ and $\operatorname{tr}_{2} A$ respectively.

\section{$5 \quad$ Trace inequalities for two by two block matrices}

Positive semidefinite $2 \times 2$ block matrices are extensively studied, such a partition yields a great deal of versatile and elegant matrix inequalities; see, e.g., [13, 18, 21, 12] for details. Recently, Kittaneh and Lin [18] (or see [26]) proved the following trace inequalities.

Theorem 5.1 [18, 26] Let $\left[\begin{array}{cc}A & B \\ B^{*} & C\end{array}\right] \in \mathbb{M}_{2}\left(\mathbb{M}_{k}\right)$ be positive semidefinite. Then

$$
\operatorname{tr} A \operatorname{tr} C-\operatorname{tr} B^{*} \operatorname{tr} B \geq\left|\operatorname{tr} A C-\operatorname{tr} B^{*} B\right|
$$

and

$$
\operatorname{tr} A \operatorname{tr} C+\operatorname{tr} B^{*} \operatorname{tr} B \geq \operatorname{tr} A C+\operatorname{tr} B^{*} B
$$

In this section, we present some inequalities related to trace for $2 \times 2$ block matrices, which are slight extensions of the result of Kittaneh and Lin. We now need to introduce some notations. Let $\otimes^{r} A:=A \otimes \cdots \otimes A$ be the $r$-fold tensor power of $A$.

Theorem 5.2 Let $\left[\begin{array}{cc}A & B \\ B^{*} & C\end{array}\right] \in \mathbb{M}_{2}\left(\mathbb{M}_{k}\right)$ be positive semidefinite. Then for $r \in \mathbb{N}^{*}$,

$$
(\operatorname{tr} A \operatorname{tr} C)^{r}-\left(\operatorname{tr} B^{*} \operatorname{tr} B\right)^{r} \geq\left|(\operatorname{tr} A C)^{r}-\left(\operatorname{tr} B^{*} B\right)^{r}\right|,
$$

and

$$
(\operatorname{tr} A \operatorname{tr} C)^{r}+\left(\operatorname{tr} B^{*} \operatorname{tr} B\right)^{r} \geq(\operatorname{tr} A C)^{r}+\left(\operatorname{tr} B^{*} B\right)^{r}
$$


Proof. Note that $\left[\begin{array}{ll}\otimes^{r} A & \otimes^{r} B \\ \otimes^{r} B^{*} & \otimes^{r} C\end{array}\right]$ is a principal submatrix of $\otimes^{r}\left[\begin{array}{cc}A & B \\ B^{*} & C\end{array}\right]$. Thus

$$
\left[\begin{array}{ll}
\otimes^{r} A & \otimes^{r} B \\
\otimes^{r} B^{*} & \otimes^{r} C
\end{array}\right]
$$

is again positive semidefinite. By applying Theorem 5.1 to this block matrix, we get

$$
\left|\operatorname{tr}\left(\otimes^{r} A\right)\left(\otimes^{r} C\right)-\operatorname{tr}\left(\otimes^{r} B^{*}\right)\left(\otimes^{r} B\right)\right| \leq \operatorname{tr} \otimes^{r} A \operatorname{tr} \otimes^{r} C-\operatorname{tr} \otimes^{r} B^{*} \operatorname{tr} \otimes^{r} B,
$$

and

$$
\operatorname{tr}\left(\otimes^{r} A\right)\left(\otimes^{r} C\right)+\operatorname{tr}\left(\otimes^{r} B^{*}\right)\left(\otimes^{r} B\right) \leq \operatorname{tr} \otimes^{r} A \operatorname{tr} \otimes^{r} C+\operatorname{tr} \otimes^{r} B^{*} \operatorname{tr} \otimes^{r} B
$$

Invoking the well-known facts [35, Chapter 2]: $\left(\otimes^{r} X\right)\left(\otimes^{r} Y\right)=\otimes^{r}(X Y)$ and $\operatorname{tr}\left(\otimes^{r} X\right)=$ $(\operatorname{tr} X)^{r}$, the desired inequalities follow immediately.

Remark. Theorem 5.2 was proved in the first version of our manuscript (announced on March 10, 2020, arXiv: 2003.04520v1). We remark that this result was recently and independently rediscovered by $\mathrm{Fu}$ and Gumus in [12] using a quite different method.

Let $e_{t}(X)$ denote the $t$-th elementary symmetric function of the eigenvalues of the square matrix $X$.

$$
e_{t}(X):=\sum_{1 \leq i_{1}<i_{2}<\cdots<i_{t} \leq n} \prod_{j=1}^{t} \lambda_{i_{j}}(X) .
$$

In particular, we know that $e_{1}(X)=\operatorname{tr}(X)$. We can get the following theorem.

Theorem 5.3 Let $\left[\begin{array}{cc}A & B \\ B^{*} & C\end{array}\right] \in \mathbb{M}_{2}\left(\mathbb{M}_{k}\right)$ be positive semidefinite. Then for $t \in\{1,2, \ldots, k\}$,

$$
e_{t}(A) e_{t}(C)-e_{t}\left(B^{*}\right) e_{t}(B) \geq\left|e_{t}(A C)-e_{t}\left(B^{*} B\right)\right|,
$$

and

$$
e_{t}(A) e_{t}(C)+e_{t}\left(B^{*}\right) e_{t}(B) \geq e_{t}(A C)+e_{t}\left(B^{*} B\right)
$$

Proof. The first inequality can be found in [18, Corollary 2.7]. We next give the outline of the proof of the second one. Note that $\left[\begin{array}{ll}\otimes^{t} A & \otimes^{t} B \\ \otimes^{t} B^{*} & \otimes^{t} C\end{array}\right]$ is positive semidefinite. By restricting this block matrix to the symmetric class of tensor product (see, e.g., [3, pp. 16-20]), we know that

$$
\left[\begin{array}{ll}
\wedge^{t} A & \wedge^{t} B \\
\wedge^{t} B^{*} & \wedge^{t} C
\end{array}\right]
$$

is still positive semidefinite. Note that $e_{t}(X)=\operatorname{tr}\left(\wedge^{t} X\right)$ and $\left(\wedge^{t} X\right)\left(\wedge^{t} Y\right)=\wedge^{t}(X Y)$. Applying Theorem 5.1 to this block matrix yields the required result. 
Let $s_{t}(X)$ be the $t$-th complete symmetric polynomial of eigenvalues of $X$, i.e.,

$$
s_{t}(X):=\sum_{1 \leq i_{1} \leq i_{2} \leq \cdots \leq i_{t} \leq n} \prod_{j=1}^{t} \lambda_{i_{j}}(X) .
$$

Clearly, we have $s_{1}(X)=\operatorname{tr}(X)$. We can get the following slight extension similarly.

Theorem 5.4 Let $\left[\begin{array}{cc}A & B \\ B^{*} & C\end{array}\right] \in \mathbb{M}_{2}\left(\mathbb{M}_{k}\right)$ be positive semidefinite. Then fort $\in\{1,2, \ldots, k\}$,

$$
s_{t}(A) s_{t}(C)-s_{t}\left(B^{*}\right) s_{t}(B) \geq\left|s_{t}(A C)-s_{t}\left(B^{*} B\right)\right|,
$$

and

$$
s_{t}(A) s_{t}(C)+s_{t}\left(B^{*}\right) s_{t}(B) \geq s_{t}(A C)+s_{t}\left(B^{*} B\right)
$$

Proof. Note that $\left[\begin{array}{ll}\otimes^{t} A & \otimes^{t} B \\ \otimes^{t} B^{*} & \otimes^{t} C\end{array}\right]$ is positive semidefinite. By restricting this block matrix to the symmetric class of tensor product (see, e.g., [3, pp. 16-20]), we know that

$$
\left[\begin{array}{ll}
\mathrm{V}^{t} A & \mathrm{~V}^{t} B \\
\mathrm{~V}^{t} B^{*} & \vee^{t} C
\end{array}\right]
$$

is still positive semidefinite. Similarly, we know that $\operatorname{tr}\left(\vee^{t} X\right)=s_{t}(X)$ and $\left(\vee^{t} X\right)\left(\vee^{t} Y\right)=$ $\vee^{t}(X Y)$. Applying Theorem 5.1 to this block matrix leads to the desired result.

\section{Acknowledgments}

This paper is dedicated to Prof. Weijun Liu (Central South University) on his 60th birthday, October 22 of the lunar calendar in 2021. I would like to thank Prof. Yuejian Peng for reading carefully through an earlier version of this paper. This work was supported by NSFC (Grant No. 11931002).

\section{References}

[1] K.M.R. Audenaert, Subadditivity of $q$-entropies for $q>1$, J. Math. Phys. 48 (2007), no. 8, 083507.

[2] T. Ando, Matrix inequalities involving partial traces, ILAS Conference, 2014.

[3] R. Bhatia, Matrix Analysis, GTM 169, Springer-Verlag, New York, 1997.

[4] R. Bhatia, Positive Definite Matrices, Princeton University Press, Princeton, 2007. 
[5] A. Desenyei, D. Petz, Partial subadditivity of entropies, Linear Algebra Appl. 439 (2013) 3297-3305.

[6] D. Choi, Inequalities related to trace and determinant of positive semidefinite block matrices, Linear Algebra Appl. 532 (2017) 1-7.

[7] D. Choi, Inequalities about partial transpose and partial traces, Linear Multilinear Algebra 66 (2018) 1619-1625.

[8] D. Choi, T.-Y. Tam, P. Zhang, Extension of Fischer's inequality, Linear Algebra Appl. 569 (2019) 311-322.

[9] M. Fiedler, T.L. Markham, On a theorem of Everitt, Thompson and de Pillis, Math. Slovaca 44 (1994) 441-444.

[10] X. Fu, P.-S. Lau, T.-Y. Tam, Linear maps of positive partial transpose matrices and singular value inequalities, Math. Inequal. Appl. 23 (4) (2020) 1459-1468.

[11] X. Fu, P.-S. Lau, T.-Y. Tam, Inequalities on partial traces of positive semidefinite block matrices, Canad. Math. Bull. 64 (4) (2021) 964-969.

[12] X. Fu, M. Gumus, Trace inequalities involving positive semidefinite block matrices, Linear Multilinear Algebra (2021) https://doi.org/10.1080/03081087.2021.1942418.

[13] M. Gumus, J. Liu, S. Raouafi, T.-Y. Tam, Positive semi-definite $2 \times 2$ block matrices and norm inequalities, Linear Algebra Appl. 551 (2018) 83-91.

[14] R.A. Horn, C.R. Johnson, Matrix Analysis, 2nd ed., Cambridge University Press, Cambridge, 2013.

[15] A. Jenčová, M.B. Ruskai, A unified treatment of convexity of relative entropy and related trace functions, with conditions for equality, Rev. Math. Phys. 22 (2010) 1099-1121.

[16] X. Jiang, Y. Zheng, X. Chen, Extending a refinement of Kotelianskii's inequality, Linear Algebra Appl. 574 (2019) 252-261.

[17] L. Kuai, An extension of the Fiedler-Markham determinant inequality, Linear Multilinear Algebra 66 (2018) 547-553.

[18] F. Kittaneh, M. Lin, Trace inequalities for positive semidefinite block matrices, Linear Algebra Appl. 524 (2017) 153-158.

[19] E.-Y. Lee, The off-diagonal block of a PPT matrix, Linear Algebra Appl. 486 (2015), 449-453. 
[20] Y. Li, L. Feng, Z. Huang, W. Liu, Inequalities regarding partial trace and partial determinant, Math. Inequal. Appl. 23 (2020) 477-485.

[21] Y. Li, Y. Huang, L. Feng, W. Liu, Some applications of two completely copositive maps, Linear Algebra Appl. 590 (2020) 124-132.

[22] Y. Li, W. Liu, Y. Huang, A new matrix inequality involving partial traces, Operators and Matrices 15 (2021), no. 3, 1189-1199.

[23] Y. Li, L. Feng, An Oppenheim type determinantal inequality for the Khatri-Rao product, Operators and Matrices 15 (2021), no. 2, 693-701.

[24] Y. Li, Y. Peng, An Oppenheim type inequality for positive definite block matrices, Linear Multilinear Algebra (2021) https://doi.org/10.1080/03081087.2021.1882370.

[25] M. Lin, An Oppenheim type inequality for a block Hadamard product, Linear Algebra Appl. 452 (2014) 1-6.

[26] M. Lin, A completely PPT map, Linear Algebra Appl. 459 (2014) 404-410.

[27] M. Lin, Inequalities related to $2 \times 2$ block PPT matrices, Operators and Matrices 9 (2015), no.4, 917-924.

[28] M. Lin, Extension of a result of Haynsworth and Hartfiel, Arch. Math. 104 (2015) 93-100.

[29] M. Lin, A treatment of a determinant inequality of Fiedler and Markham, Czech. Math. J. 66 (2016) 737-742.

[30] M. Lin, A determinantal inequality involving partial traces, Canad. Math. Bull. 59 (2016) 585-591.

[31] M. Lin, P. Zhang, Unifying a result of Thompson and a result of Fiedler and Markham on block positive definite matrices, Linear Algebra Appl. 533 (2017) $380-385$.

[32] D. Petz, Quantum Information Theory and Quantum Statistics. Theoretical and Mathematical Physics, Springer, Berlin, 2008.

[33] A.E. Rastegin, Relations for symmetric norms and anti-norms before and after partial trace, J. Stat. Phys. 148 (2012) 1040-1053.

[34] J. Yang, L. Lu, Z. Chen, Schatten $q$-norms and determinantal inequalities for matrices with numerical ranges in a sector, Linear Multilinear Algebra 67 (2019) $221-227$. 
[35] X. Zhan, Matrix Theory, Graduate Studies in Mathematics, vol. 147, Amer. Math. Soc., Providence, RI, 2013.

[36] F. Zhang, Matrix Theory: Basic Results and Techniques, 2nd ed., Springer, New York, 2011.

[37] F. Zhang, Positivity of matrices with generalized matrix functions. Acta Math. Sin. (Engl. Ser.) 28 (2012) 1779-1786.

[38] P. Zhang, Extension of Matic's results, Linear Algebra Appl. 486 (2015) 328-334.

[39] P. Zhang, On some inequalities related to positive block matrices, Linear Algebra Appl. 576 (2019) 258-267. 